\title{
Parametric bootstrap under model mis-specification
}

\author{
H. Y. Kevin Lu, G. Alastair Young ${ }^{1, *}$ \\ Department of Mathematics, Imperial College London, London, SW7 2AZ, UK
}

\begin{abstract}
Under model correctness, highly accurate inference on a scalar interest parameter in the presence of a nuisance parameter can be achieved by several routes, among them considering the bootstrap distribution of the signed root likelihood ratio statistic. The context of model mis-specification is considered and inference based on a robust form of the signed root statistic is discussed in detail. Stability of the distribution of the statistic allows accurate inference, outperforming that based on first-order asymptotic approximation, by considering the bootstrap distribution of the statistic under the incorrectly assumed distribution. Comparisons of this simple approach with alternative analytic and non-parametric inference schemes are discussed.
\end{abstract}

Keywords:

Asymptotic approximation, model mis-specification, non-parametric inference, parametric bootstrap, resampling, signed root likelihood ratio statistic

\section{Introduction}

Let $Y=\left\{Y_{1}, \ldots, Y_{n}\right\}$ be a random sample of size $n$, from a distribution assumed to have probability density function $f(y ; \eta)$, with $\eta=(\psi, \lambda)$, where $\psi$ is a scalar interest parameter and $\lambda$ a nuisance parameter, possibly vectorvalued. Consider testing the null hypothesis $H_{0}: \psi=\psi_{0}$, with $\psi_{0}$ specified, against a one-sided alternative of the form $H_{1}: \psi<\psi_{0}$ or $H_{1}: \psi>\psi_{0}$.

\footnotetext{
${ }^{*}$ Corresponding author.

Email addresses: kevin.lu02@imperial.ac.uk (H. Y. Kevin Lu), alastair.young@imperial.ac.uk (G. Alastair Young)

${ }^{1}$ Telephone number: $+44(0) 2075948560$
} 
Let $l(\eta) \equiv l(\eta ; Y)$ be the log-likelihood for $\eta$ based on $Y$. Also, denote by $\hat{\eta}=(\hat{\psi}, \hat{\lambda})$ the overall maximum likelihood estimator of $\eta$, and by $\hat{\lambda}_{\psi}$ the constrained maximum likelihood estimator of $\lambda$, for a given fixed value of $\psi$.

Inference may be based on the signed square root likelihood ratio statistic, defined by

$$
R \equiv R\left(\psi_{0}\right)=\operatorname{sgn}\left(\hat{\psi}-\psi_{0}\right)\left[2\left\{l(\hat{\psi}, \hat{\lambda})-l\left(\psi_{0}, \hat{\lambda}_{0}\right)\right\}\right]^{1 / 2},
$$

where $\operatorname{sgn}(x)=-1$ if $x<0,=0$ if $x=0$ and $=1$ if $x>0$, and $\hat{\lambda}_{0}=\hat{\lambda}_{\psi_{0}}$. Under $H_{0}, R\left(\psi_{0}\right)$ is asymptotically distributed according to the standard normal distribution $N(0,1)$, provided the assumed parametric distribution is correct. The level of error of the $N(0,1)$ approximation to the sampling distribution of $R\left(\psi_{0}\right)$ is of the first-order, $O\left(n^{-1 / 2}\right)$ in the sample size $n$.

Two main approaches emerge (Young, 2009) to reduce this level of error, to the third-order, $O\left(n^{-3 / 2}\right)$ : analytic adjustment of the statistic $R$, and replacement of the $N(0,1)$ approximation by a bootstrap estimate of the distribution of the statistic. A key form of analytically adjusted statistic is Barndorff-Nielsen's $R^{*}$ statistic (Barndorff-Nielsen, 1986), which is of the form $R^{*}=R+\log (U / R) / R$, in terms of an analytic adjustment quantity $U$. DiCiccio et al. (2001) and Lee \& Young (2005) considered inference based on the bootstrap distribution obtained by considering the distribution of $R\left(\psi_{0}\right)$ under sampling from the density $f\left(y ; \psi_{0}, \hat{\lambda}_{0}\right)$. Both of these third-order accurate inference procedures are observed in many situations to achieve spectacularly low levels of error even in small sample settings.

Concern here is with the stated inference problem in circumstances when $Y$ is a random sample from a density $g(y)$ which does not belong to the assumed parametric family of densities $f(y ; \psi, \lambda)$. Specific consideration is given to the following formulation of the inference problem under model misspecification, as described, for example, by Kent (1982) and Stafford (1996). Let $\eta(g)=\{\psi(g), \lambda(g)\}$ minimise the Kullback-Leibler distance between $g(y)$ and $f(y ; \eta)$, given by $\int \log \{g(y) / f(y ; \eta)\} g(y) d y$. Then consider testing $H_{0}: \psi=\psi_{0}$, with $\psi_{0}=\psi(g)$. Such an inference problem is natural whenever $\psi(g)$ has a direct interpretation under the true $g(y)$, for example as an expected value. In these circumstances, the statistic $R\left(\psi_{0}\right)$ is asymptotically distributed as $N(0, v)$, where $v \equiv v(g) \neq 1$ in general. It has been suggested (Stafford, 1996: see also related work by Viraswami \& Reid, 1996, 1998) that the signed root likelihood ratio statistic $R\left(\psi_{0}\right)$ be 'robustified' by rescaling, through construction of a statistic of the form $R^{\prime} \equiv R^{\prime}\left(\psi_{0}\right)=R / \sqrt{\hat{v}}$, where 
$\hat{v}$ is an empirical estimate, constructed from $Y$, of the asymptotic variance $v$. Such a modified statistic $R^{\prime}\left(\psi_{0}\right)$ is asymptotically distributed as standard normal under $H_{0}$. Again, the level of error of a $N(0,1)$ approximation to the sampling distribution is of order $O\left(n^{-1 / 2}\right)$.

The purpose here is to examine closely the use of the statistic $R^{\prime}$ for inference, expanding on comments made by Lu \& Young (2010). The objective is to stress the following methodological conclusions. When there is no model mis-specification, highly accurate inference can be achieved by bootstrapping the distribution of $R^{\prime}$ : since $R^{\prime}$ is asymptotically distributed as $N(0,1)$ it follows directly from Lee \& Young $(2005)$ that this procedure achieves the same third-order accuracy as inference based on normal approximation to the distribution of Barndorff-Nielsen's $R^{*}$ statistic. Under model mis-specification, $R^{*}$ is non-robust and $N(0,1)$ approximation to its sampling distribution does not achieve a test with the correct asymptotic level. However, the parametric bootstrap procedure, which again samples from the (incorrect) density $f\left(y ; \psi_{0}, \hat{\lambda}_{0}\right)$ is shown to yield accurate inference. Though in principle the level of error, $O\left(n^{-1 / 2}\right)$, is no better than that offered by normal approximation to the sampling distribution, in practice the bootstrap procedure substantially outperforms normal approximation. The key to this property is that the distribution of $R^{\prime}$ typically does not depend much on the true density underlying the sample data $Y$, but converges rather slowly to its asymptotic limit. Therefore, using the sampling distribution of $R^{\prime}$ under the wrong density $f\left(y ; \psi_{0}, \hat{\lambda}_{0}\right)$ as a surrogate for its distribution under the true density $g$ is often a rather accurate estimation procedure, in particular for small $n$.

These observations, together with empirical comparisons between the parametric bootstrap procedure and non-parametric alternatives, suggest strongly that in the inference problem being considered the most effective approach is based on the distribution of the modified statistic $R^{\prime}$, under sampling from the density $f\left(y ; \psi_{0}, \hat{\lambda}_{0}\right)$. This procedure yields highly accurate inference, with the same low levels of error as obtained by use of the $R^{*}$ statistic, when the parametric assumption is correct, while protecting against model mis-specification. The analysis demonstrates, in particular, that in the latter setting the parametric bootstrap procedure, based on the wrong distribution, will often outperform the asymptotic method based on $N(0,1)$ approximation to the distribution of $R^{\prime}$. This indicates that higher levels of accuracy than obtained by first-order asymptotic methods will often be achievable in this setting. 


\section{Asymptotic distribution of $\boldsymbol{R}^{\prime}$}

Use is made of the notation, the stochastic expansion of $R$ and the expression for $\hat{v}$ provided in Stafford (1996) to find the cumulants of the robust statistic $R^{\prime}$. These cumulants are seen to be asymptotically the same as the cumulants of $N(0,1)$, thereby showing that the asymptotic distribution of $R^{\prime}$ is standard normal.

Suppose $\eta=\left(\eta_{1}, \eta_{2}, \ldots, \eta_{d}\right)$, where $\eta_{1}=\psi$ is the scalar interest parameter, $\left(\eta_{2}, \ldots, \eta_{d}\right)=\lambda$ is the vector nuisance parameter and $d$ is the dimension of $\eta$. Let $l_{i}=l_{i}(\eta)=\log f\left(y_{i} ; \eta\right)$ be the log-likelihood of the $i^{\text {th }}$ observation, and let $l_{i ; s}=\frac{\partial}{\partial \eta_{s}} l_{i}(\eta), l_{i ; s t}=\frac{\partial^{2}}{\partial \eta_{s} \partial \eta_{t}} l_{i}(\eta)$ be the partial derivatives of the log-likelihood for the $i^{\text {th }}$ observation, $s, t=1, \ldots, d$.

Denote by $\eta_{0}=\left(\psi_{0}, \lambda_{0}\right)$ the value of $\eta$ which minimises the KullbackLeibler distance, as described in the previous section.

In the following definitions and derivations, it is not necessary to assume whether the true distribution is mis-specified by $f$ or not: the same asymptotic results hold true for both cases of $g=f$ and $g \neq f$.

Define

$$
\begin{gathered}
I_{s t} \equiv I_{s t}\left(\eta_{0}\right)=\left.E_{g(y)}\left[l_{1 ; s t}\right]\right|_{\eta=\eta_{0}}, \\
I_{s, t} \equiv I_{s, t}\left(\eta_{0}\right)=\left.E_{g(y)}\left[l_{1 ; s} l_{1 ; t}\right]\right|_{\eta=\eta_{0}}
\end{gathered}
$$

and

$$
Z_{s} \equiv Z_{s}\left(\eta_{0}\right)=\left.\frac{1}{\sqrt{n}} \sum_{i=1}^{n} l_{i ; s}\right|_{\eta=\eta_{0}},
$$

where $E_{g(y)}[$.$] denotes the expectation with respect to g(y)$.

Further define a $d \times d$ matrix $A$ with components $I_{s t}$ and denote the components of the $d \times d$ matrix $-A^{-1}$ by $I^{s t}$, where $A^{-1}$ is the usual matrix inverse of $A$. Denote by $\hat{I}_{s t} \equiv \hat{I}_{s t}(\hat{\eta})=\left.\frac{1}{n} \sum_{i=1}^{n} l_{i ; s t}\right|_{\eta=\hat{\eta}}$ and $\hat{I}_{s, t} \equiv \hat{I}_{s, t}(\hat{\eta})=$ $\left.\frac{1}{n} \sum_{i=1}^{n} l_{i ; s} l_{i ; t}\right|_{\eta=\hat{\eta}}$ estimates of $I_{s t}$ and $I_{s, t}$ respectively. An estimate of $A$ is $\hat{A}$, obtained by the replacement of $I_{s t}$ in $A$ by $\hat{I}_{s t}$. Then, $\hat{I}^{s t}$, which is an estimate of $I^{s t}$, can be read off from the corresponding $(s, t)$-entry of the matrix $-\hat{A}^{-1}$.

Using these definitions, an expansion of $R^{\prime}$ to error of order $O_{p}\left(n^{-1 / 2}\right)$ can be derived and is

$$
R^{\prime} \equiv R / \sqrt{\hat{v}}=Z^{\psi} / \sqrt{v}+O_{p}\left(n^{-1 / 2}\right),
$$

where $Z^{\psi}=\sum_{s=1}^{d} I^{1 s} Z_{s}, v=\sum_{s=1}^{d} \sum_{t=1}^{d} I^{1 s} I_{s, t} I^{t 1} / I^{11}$ and $\hat{v}=\sum_{s=1}^{d} \sum_{t=1}^{d} \hat{I}^{1 s} \hat{I}_{s, t} \hat{I}^{t 1} / \hat{I}^{11}=v+O_{p}\left(n^{-1 / 2}\right)$. 
Denote the $i^{\text {th }}$ cumulant of $R^{\prime}$ by $\kappa_{i}^{R^{\prime}}$ and let $X=\frac{Z^{\psi}}{\sqrt{v}}$. The first four cumulants of $R^{\prime}$ can be calculated as

$$
\begin{aligned}
\kappa_{1}^{R^{\prime}}= & E[X]+O\left(n^{-1 / 2}\right)=O\left(n^{-1 / 2}\right) \\
\kappa_{2}^{R^{\prime}}= & E\left[X^{2}\right]-E[X]^{2}+O\left(n^{-1 / 2}\right)=1+O\left(n^{-1 / 2}\right) \\
\kappa_{3}^{R^{\prime}}= & E\left[X^{3}\right]-3 E\left[X^{2}\right] E[X]+E[X]^{3}+O\left(n^{-1 / 2}\right)=O\left(n^{-1 / 2}\right) \\
\kappa_{4}^{R^{\prime}}= & E\left[X^{4}\right]-4 E\left[X^{3}\right] E[X]-3 E\left[X^{2}\right]^{2}+12 E\left[X^{2}\right] E[X]^{2}-6 E[X]^{4} \\
& +O\left(n^{-1 / 2}\right)=O\left(n^{-1 / 2}\right) .
\end{aligned}
$$

The fifth and higher cumulants can be calculated to be 0 to error of order $O\left(n^{-1 / 2}\right)$ or smaller. These cumulants show that the distribution of $R^{\prime}$ is asymptotically $N(0,1)$ whether $g$ is mis-specified by $f$ or not. Explicit evaluation of the leading terms in these cumulant expansions allows construction of analytic approximations of Edgeworth and saddlepoint types to the distribution of $R^{\prime}$. However, in practice, since $g$ is unknown, it is necessary to replace cumulants by empirical estimates. Extensive investigations in the first author's Ph.D. thesis indicate that such analytic approximations yield poor distribution estimates, unless the sample size $n$ is very large, or the true cumulants of $R^{\prime}$ are known. These findings agree with remarks made by Viraswami \& Reid $(1996,1998)$. The potential, therefore, for analytic approaches to yield high levels of accuracy under the model mis-specification formulation seems strictly limited. Therefore focus here is on investigation of improvements over the $N(0,1)$ approximation to the distribution that may be obtainable with small sample sizes by the parametric bootstrap route.

\section{Parametric bootstrap method}

Given the assumed density $f(y ; \psi, \lambda)$, to approximate the distribution under $H_{0}: \psi=\psi_{0}$ of $R^{\prime}, P\left(R^{\prime} \leq r^{\prime}\right)$, where $r^{\prime}$ is the value of $R^{\prime}$ based on $Y$, a parametric bootstrap method uses the following procedure:

Step 1 Generate $\tilde{B}$ datasets $\tilde{Y}^{(1)}, \ldots, \tilde{Y}^{(\tilde{B})}$, each of which consists of $n$ independent, identically distributed values simulated from $f\left(y ; \psi_{0}, \hat{\lambda}_{0}\right)$, where $\left(\psi_{0}, \hat{\lambda}_{0}\right)$ is the constrained MLE of $(\psi, \lambda)$ evaluated using $Y$ and fixed $\psi=\psi_{0}$.

Step 2 Calculate the values of the statistic $R^{\prime}, \tilde{r}_{1}^{\prime}, \ldots, \tilde{r}_{\tilde{B}}^{\prime}$, from each generated sample $\tilde{Y}^{(1)}, \ldots, \tilde{Y}^{(\tilde{B})}$. 
Step 3 Find the proportion of the values $\tilde{r}^{\prime}$ calculated in Step 2 which are smaller than or equal to $r^{\prime}$.

So, $P\left(R^{\prime} \leq r^{\prime}\right)$ is estimated by the proportion $\hat{p}=\frac{1}{\tilde{B}} \sum_{j=1}^{\tilde{B}} I\left(\tilde{r}_{j}^{\prime} \leq r^{\prime}\right)$, where $I(Q)$ is an indicator function taking the value 1 if the statement $Q$ is true and the value 0 otherwise.

This approximation may be called the null hypothesis bootstrap procedure, and has error of order $O\left(n^{-3 / 2}\right)$ when $g=f$. An alternative version of the approximation uses the same procedure, but $\left(\psi_{0}, \hat{\lambda}_{0}\right)$ in Step 1 is replaced by the full MLE $(\hat{\psi}, \hat{\lambda})$. Using $(\hat{\psi}, \hat{\lambda})$ in the approximation results in an error of magnitude $O\left(n^{-1}\right)$ and hence this version is generally not used in practice. Detailed analysis of both of these bootstrap procedures under model correctness can be found in Lee \& Young (2005).

As described, the null hypothesis bootstrap procedure approximates the distribution of $R^{\prime}$ to error of magnitude $O\left(n^{-3 / 2}\right)$, when there is no model mis-specification. When there is a model mis-specification, it was seen in the previous section that the asymptotic distribution of $R^{\prime}$ is still $N(0,1)$ under sampling from $f \neq g$. Therefore, the approximation to the distribution of $R^{\prime}$ under sampling from the wrong $f\left(y ; \psi, \hat{\lambda}_{0}\right)$ is asymptotically valid. However, it only yields an error of magnitude $O\left(n^{-1 / 2}\right)$, which is the same theoretical order as the normal approximation. In practice, this parametric bootstrap procedure may be expected to outperform $N(0,1)$ approximation if the Kullback-Leibler distance between the true density $g$ and the assumed $f$ is small, or if the distribution of $R^{\prime}$ does not depend much on $g$.

\section{Non-parametric bootstrap methods}

In the parametric null hypothesis bootstrap procedure, sampling is from the assumed distribution $f$. In contrast, the non-parametric bootstrap method samples directly from the data sample $Y$. The procedure for the nonparametric method is similar to that of the parametric method described above with some changes to Step 1.

Step 1 Generate $\tilde{B}$ datasets $\tilde{Y}^{(1)}, \ldots, \tilde{Y}^{(\tilde{B})}$, each of which consists of $n$ values simulated from $Y=\left\{Y_{1}, \ldots, Y_{n}\right\}$ with replacement, and with each point $Y_{i}$ being chosen with the probability $w_{i}$.

Step 2 Calculate $\tilde{r}_{1}^{\prime}, \ldots, \tilde{r}_{\tilde{B}}^{\prime}$ from each of $\tilde{Y}^{(1)}, \ldots, \tilde{Y}^{(\tilde{B})}$. 
Step 3 Find the proportion of $\tilde{r}^{\prime}$ calculated in Step 2 which are smaller than or equal to $r^{\prime}$.

Then the proportion $\hat{p}=\frac{1}{\tilde{B}} \sum_{j=1}^{\tilde{B}} I\left(\tilde{r}_{j}^{\prime} \leq r^{\prime}\right)$ estimates $P\left(R^{\prime} \leq r^{\prime}\right)$. In practice, it is noted that in some situations accuracy of this non-parametric scheme is improved by centring the bootstrap values generated at Step 2 to have mean 0, before calculation of the tail probability in Step 3. Though still requiring formal justification, this modification makes sense, as the null distribution of $R^{\prime}$ has, asymptotically, mean 0 . In particular, this modification is seen to improve accuracy of the non-parametric scheme noticeably in Example 4 below, with small $n$; in the other examples centring of the bootstrap distribution has less effect.

Consideration is given to two ways of specification of the weights $w_{1}, \ldots, w_{n}$ for the non-parametric sampling scheme. Full details and investigation of other possible non-parametric sampling schemes are given by the first author in his Ph.D. thesis.

It is required to resample from $Y=\left\{Y_{1}, \ldots, Y_{n}\right\}$, where each $Y_{i}$ is chosen with probability $w_{i}$, respecting the null hypothesis constraint $H_{0}: \psi=\psi_{0}$. Recall that $\left(\psi_{0}, \lambda_{0}\right)$ minimises the Kullback-Leibler distance, or equivalently, maximises

$$
T(\psi, \lambda) \equiv \int \log \{f(y ; \psi, \lambda)\} g(y) d y .
$$

Consider the distribution $\hat{g}$, which estimates the distribution $g$ and places weight $w_{i}$ on $Y_{i}$. Replacing the expectation under $g$ with that under $\hat{g}$ yields an estimate of $T$ of the form

$$
\hat{T}(\psi, \lambda)=\sum \log \left\{f\left(y_{i} ; \psi, \lambda\right)\right\} w_{i} .
$$

The resampling weights $w_{1}, \ldots, w_{n}$ are specified such that $\hat{T}(\psi, \lambda)$ is maximised at $\psi=\psi_{0}, \lambda=\tilde{\lambda}$, for some $\tilde{\lambda}$ in a neighbourhood of $\hat{\lambda}_{0}$, so that $\left.\frac{\partial}{\partial \psi} \hat{T}(\psi, \lambda)\right|_{\psi=\psi_{0}, \lambda=\tilde{\lambda}}=0,\left.\quad \frac{\partial}{\partial \lambda} \hat{T}(\psi, \lambda)\right|_{\psi=\psi_{0}, \lambda=\tilde{\lambda}}=0$. Such a procedure provides a natural comparison with the parametric null hypothesis bootstrap procedure, which replaces the unknown $\lambda_{0}$ by $\hat{\lambda}_{0}$ in the parametric sampling. Then, denoting the nuisance parameter $\lambda$ by $\left(\lambda_{1}, \ldots, \lambda_{p}\right)$, where $p=d-1$, and writing $c_{i} \equiv c_{i}\left(\psi_{0}, \tilde{\lambda}\right)=\left.\frac{\partial}{\partial \psi} \log \left\{f\left(y_{i} ; \psi, \lambda\right)\right\}\right|_{\psi=\psi_{0}, \lambda=\tilde{\lambda}}$ and $u_{i j} \equiv u_{i j}\left(\psi_{0}, \tilde{\lambda}\right)=\left.\frac{\partial}{\partial \lambda_{j}} \log \left\{f\left(y_{i} ; \psi, \lambda\right)\right\}\right|_{\psi=\psi_{0}, \lambda=\tilde{\lambda}}, j=1, \ldots, p$, it is required to find weights $w_{i}$ and $\tilde{\lambda}$ such that

$$
\sum w_{i} c_{i}=0, \sum w_{i} u_{i j}=0, j=1, \ldots, p .
$$


Another estimate of the underlying distribution, the empirical distribution $\tilde{g}$, which is obtained by placing an equal probability of $1 / n$ on each $Y_{i}$ in $D$, is used as a tool for finding the weights in $\hat{g}$. To choose the $w_{i}$, minimise the Kullback-Leibler distance between $\hat{g}$ and $\tilde{g}$, given by

$$
\sum w_{i} \log \left\{w_{i} /(1 / n)\right\}=\sum w_{i} \log w_{i}+\log n,
$$

subject to the $d$ constraints just described being satisfied. This optimisation problem is solved by standard Lagrangian techniques, to give $w_{i}$ of the form

$$
w_{i}=\exp \left(\beta c_{i}+\alpha_{1} u_{i 1}+\ldots+\alpha_{p} u_{i p}\right) /\left\{\sum_{k} \exp \left(\beta c_{k}+\alpha_{1} u_{k 1}+\ldots+\alpha_{p} u_{k p}\right)\right\}
$$

where $\beta, \alpha_{1}, \ldots, \alpha_{p}$ must be found numerically to satisfy (1).

The non-parametric procedure which involves specification of the sampling weights by (2), for some value of $\tilde{\lambda}$ for which (1) is satisfied, shall be termed 'weighted v1'. In practice, implementation of the method involves use of a grid search, in a neighbourhood of $\hat{\lambda}_{0}$, to find a value of $\tilde{\lambda}$ for which (1) is satisfied, with the sampling weights defined by (2). In the numerical examples, the grid search was initialised by seeking a solution with $\tilde{\lambda}=\hat{\lambda}_{0}$, and used small step size $\epsilon=0.01$, a maximum of 100 candidate values for $\tilde{\lambda}$ being considered. Though no attempt has been made to optimise the nonparametric scheme, it should be noted that accuracy results under repeated sampling are quite insensitive to the details of such a computational procedure. In all examples studied appropriate sampling weights $w_{i}$ fail to be found by such a computationally inexpensive search for only a small, though significant, proportion of data samples, when $n$ is small. For larger sample size, such as $n=50$, as used in some of the numerical investigations reported below, typically a solution is found with $\tilde{\lambda}=\hat{\lambda}_{0}$, and the proportion of data samples for which weights fail to be found is effectively zero.

The method described can be viewed as a special case of the methodology related to $M$-estimation detailed by Lee \& Young (2003). It follows from their theory, the conditions for which are readily verified for the current context, that this non-parametric method yields a theoretical small error rate of $O\left(n^{-3 / 2}\right)$. However, it is anticipated, from findings reported by Lee \& Young (2003), that the method is unlikely to work well in practice, in situations where the sample size $n$ is small. It is of some significance to observe in the examples generally superior accuracy from the parametric bootstrap method, even though it is of inferior theoretical accuracy under model mis-specification. 
In addition, consideration is also given to an ad hoc, simplified version of the non-parametric bootstrap method, which is referred to as 'weighted v2', and which imposes just the requirement $\left.\frac{\partial}{\partial \psi} \hat{T}(\psi, \lambda)\right|_{\psi=\psi_{0}, \lambda=\hat{\lambda}_{0}}=0$. Then the requirement is just $\sum b_{i} w_{i}=0$, where $b_{i}=\left.\frac{\partial}{\partial \psi} \log \left\{f\left(y_{i} ; \psi, \lambda\right)\right\}\right|_{\psi=\psi_{0}, \lambda=\hat{\lambda}_{0}}$. Minimising the Kullback-Leibler distance between $\hat{g}$ and $\tilde{g}$ determines that the weights for the non-parametric sampling are now given by

$$
w_{i}=\exp \left(\beta b_{i}\right) /\left\{\sum_{j} \exp \left(\beta b_{j}\right)\right\},
$$

where $\beta$ is such that $\sum b_{i} \exp \left(\beta b_{i}\right)=0$. Now it is easy to analyse circumstances where sampling weights can be found: there exists $\beta$ such that $\sum b_{i} \exp \left(\beta b_{i}\right)=0$ if there exist $i, j \in\{1, \ldots, n\}$ such that $b_{i} b_{j}<0$, a condition easy to check. The informal nature of this scheme weighted v2, however, means that it is not possible to specify a formal error rate.

In the numerical evaluations, the convention is adopted that when appropriate sampling weights cannot be found for the non-parametric schemes, the bootstrap tail probability estimate is taken as the estimate obtained by the normal approximation to the relevant statistic. As will be seen in the next section, the proportion of replications where weights cannot be found depends on $f$ and $g$. The numerical results will show further that the informal scheme weighted v2 tends actually to be superior to weighted v1.

\section{Examples}

In each of the following examples, comparisons are drawn between different approximation methods and the use of the statistics $R, R^{\prime}$ and $R^{*}$ under both model mis-specification and no model mis-specification, with emphasis on the former. When there is model mis-specification, the null hypothesis will be $H_{0}: \psi=\psi_{0}$, where $\eta_{0}=\left(\psi_{0}, \lambda_{0}\right)$ minimises the Kullback-Leibler distance between the true distribution and the assumed distribution. On the other hand, when there is no model mis-specification, the null hypothesis will also be $H_{0}: \psi=\psi_{0}$, but now $\psi_{0}$ is the parameter value of the true distribution. The alternative hypothesis will be $H_{1}: \psi<\psi_{0}$ for both cases.

In the simulations, the replication size and the bootstrap size for both parametric and non-parametric bootstrap methods will be set at $B=\tilde{B}=$ $10^{4}$. Primary interest is in accuracy properties of the methods for small sample size situations. Therefore, primary focus is on the case where the 
sample size is fixed at $n=10$, which ensures that most points in a nonparametric resampled dataset are distinct. Simulation results for sample size $n=50$ can be found in the Appendix and give a good impression of the relative accuracies of the different methods with larger sample sizes.

Approximation results, which are empirical estimates of actual sizes of hypothesis tests as determined from the series of $B$ replications, will be presented in tables. The closer the results to their respective nominal sizes, the better the approximation and the statistic used.

Example 1. Gamma v.s. Inverse Gaussian. Let $Y_{1}, \ldots, Y_{n}$ be a sample from a gamma distribution having scale parameter $b=1$ and shape parameter $c=5.5$. Suppose the sample is assumed to be from an inverse Gaussian (IG) distribution with mean $\mu$ and shape parameter $\xi$, which is the interest parameter. The probability density function of IG is taken to be $f(y ; \xi, \mu)=\left(\frac{\xi}{2 \pi y^{3}}\right)^{1 / 2} \exp \left\{-\frac{\xi}{2 \mu^{2} y}\left(y-\mu^{2}\right)\right\}, y>0$.

It can be found that $\eta_{0}=\left(\xi_{0}, \mu_{0}\right)=\left(\left[\frac{\Gamma(c-1)}{b \Gamma(c)}-\frac{1}{b c}\right]^{-1}, b c\right)$ minimises the Kullback-Leibler distance between the two distributions and therefore the null hypothesis is set to be $H_{0}: \xi=\xi_{0}$.

The overall maximum likelihood estimator (MLE) of $\eta=(\xi, \mu)$ under the assumed distribution is $\hat{\eta}=(\hat{\xi}, \hat{\mu})=\left(\left[\frac{1}{n} \sum \frac{\left(Y_{i}-\hat{\mu}\right)^{2} Y_{i}}{\hat{\mu}^{2}}\right]^{-1}, \frac{1}{n} \sum Y_{i}\right)$, and the constrained MLE of $\eta$ is $\hat{\eta}_{0}=\left(\xi_{0}, \frac{1}{n} \sum Y_{i}\right)$. Table 1 displays results for

Table 1: Actual sizes of test under model mis-specification: Gamma v.s. IG, $n=10$, $H_{0}: \xi=\xi_{0}$. Table entries are percentage.

\begin{tabular}{ccccccccc} 
Nominal size & $1 \%$ & $2.5 \%$ & $5 \%$ & $10 \%$ & $90 \%$ & $95 \%$ & $97.5 \%$ & $99 \%$ \\
\hline$\Phi(R)$ & 1.87 & 3.12 & 4.78 & 7.99 & 75.55 & 84.81 & 90.88 & 95.50 \\
$\Phi\left(R^{\prime}\right)$ & 2.01 & 3.36 & 5.24 & 8.60 & 69.24 & 77.24 & 83.26 & 88.62 \\
$\Phi\left(R^{*}\right)$ & 2.92 & 4.94 & 7.78 & 12.54 & 86.04 & 92.33 & 95.98 & 98.17 \\
Null hyp., $R^{\prime}$ & 1.07 & 2.59 & 5.25 & 10.23 & 87.10 & 93.29 & 96.54 & 98.50 \\
W. v1, $R^{\prime}(2.84 \%)$ & 0.89 & 2.33 & 4.55 & 9.49 & 72.94 & 79.82 & 84.63 & 88.73 \\
W. v2, $R^{\prime}(5.55 \%)$ & 1.03 & 2.59 & 4.89 & 9.69 & 82.98 & 89.16 & 91.62 & 93.08
\end{tabular}

normal approximations to $R, R^{\prime}$ and $R^{*}$, in the first, second and third rows respectively, and approximations using $R^{\prime}$ by the null hypothesis bootstrap procedure (Null hyp.), the weighted resampling method version 1 (W. v1), and version 2 (W. v2), with results in the fourth, fifth and sixth rows. The percentage figures in parentheses in the table here and in the rest of the paper 
give the proportion of the $B$ simulations where resampling weights cannot be determined, in which case standard normal approximation is used.

It can be seen from the table that the null hypothesis bootstrap procedure yields the best approximations, giving estimated sizes which are closest to the nominal sizes, with the next best method in terms of accuracy being weighted $\mathrm{v} 2$. The failure rate of weighted $\mathrm{v} 1$ is about half that of weighted v2, with the accuracy of weighted v2 somewhat better than that of weighted v1. The reason why the normal approximations to the distributions of the non-robust statistics $R$ and $R^{*}$ are better than that for the robust statistic $R^{\prime}$ is because the distribution of $R^{\prime}$ at small sample size $n=10$ is quite different from $N(0,1)$ in this setting, even though $R^{\prime}$ is asymptotically standard normal $N(0,1)$, while $R$ and $R^{*}$ are not. When there is no model mis-specification, so

Table 2: Actual sizes of test under no model mis-specification: IG v.s. IG, $n=10$, $H_{0}: \xi=\xi_{0}=2$. Table entries are percentage.

\begin{tabular}{ccccccccc} 
Nominal size & $1 \%$ & $2.5 \%$ & $5 \%$ & $10 \%$ & $90 \%$ & $95 \%$ & $97.5 \%$ & $99 \%$ \\
\hline$\Phi(R)$ & 0.36 & 1.09 & 2.29 & 5.07 & 80.75 & 88.38 & 93.39 & 96.87 \\
$\Phi\left(R^{\prime}\right)$ & 1.96 & 3.44 & 5.14 & 8.25 & 73.53 & 81.18 & 86.63 & 91.06 \\
$\Phi\left(R^{*}\right)$ & 0.97 & 2.37 & 4.84 & 9.73 & 89.29 & 94.72 & 97.23 & 98.87 \\
Null hyp., $R^{\prime}$ & 1.15 & 2.74 & 5.11 & 10.14 & 89.61 & 94.58 & 97.39 & 98.98 \\
W. v1, $R^{\prime}(0.50 \%)$ & 1.30 & 2.91 & 5.24 & 10.24 & 77.91 & 83.75 & 88.24 & 92.06 \\
W. v2, $R^{\prime}(4.22 \%)$ & 1.25 & 2.89 & 5.07 & 9.96 & 86.11 & 91.90 & 93.70 & 94.66
\end{tabular}

that $Y_{1}, \ldots, Y_{n}$ are indeed from an inverse Gaussian distribution with $\xi_{0}=2$ and $\mu_{0}=1$, the approximations given in Table 2 show that both the normal approximation to $R^{*}$ and the null hypothesis bootstrap procedure applied to $R^{\prime}$ give similarly good approximations, as expected. In addition, these two methods perform much better than the normal approximation to $R$ or $R^{\prime}$, and the non-parametric alternatives. Note that all methods give noticeably more accurate approximations than seen in the model mis-specification context.

Example 2. F-distribution v.s. Log-normal. Let $Y_{1}, \ldots, Y_{n}$ follow an Fdistribution with density $g$ having degrees of freedom $d_{1}=1$ and $d_{2}=2$. Suppose the sample is assumed to be from a log-normal (LN) distribution with mean-log parameter $\mu$ and variance-log parameter $\sigma^{2}$. The interest parameter is the standard deviation log parameter $\sigma$, the positive square root of the variance-log, and the probability density function of $\operatorname{LN}$ is $f(y ; \sigma, \mu)=$ $\frac{1}{y \sigma \sqrt{2 \pi}} \exp \left\{-\frac{(\log y-\mu)^{2}}{2 \sigma^{2}}\right\}, y>0$. 
Table 3: Actual sizes of test under model mis-specification: F v.s. LN, $n=10, H_{0}: \sigma=\sigma_{0}$. Table entries are percentage.

\begin{tabular}{ccccccccc} 
Nominal size & $1 \%$ & $2.5 \%$ & $5 \%$ & $10 \%$ & $90 \%$ & $95 \%$ & $97.5 \%$ & $99 \%$ \\
\hline$\Phi(R)$ & 6.94 & 12.80 & 19.28 & 29.58 & 90.65 & 93.87 & 95.68 & 97.12 \\
$\Phi\left(R^{\prime}\right)$ & 14.48 & 20.01 & 26.04 & 34.49 & 90.36 & 93.78 & 95.75 & 97.34 \\
$\Phi\left(R^{*}\right)$ & 2.88 & 6.21 & 10.94 & 18.01 & 86.39 & 90.76 & 93.76 & 95.90 \\
Null hyp., $R^{\prime}$ & 2.25 & 5.19 & 8.86 & 15.89 & 88.76 & 93.94 & 96.57 & 98.55 \\
W. v1, $R^{\prime}(2.13 \%)$ & 9.54 & 14.64 & 19.94 & 27.11 & 88.87 & 94.09 & 96.83 & 98.53 \\
W. v2, $R^{\prime}(7.78 \%)$ & 9.66 & 11.80 & 15.06 & 20.75 & 89.22 & 93.86 & 96.50 & 98.16
\end{tabular}

Table 4: Actual sizes of test under no model mis-specification: LN v.s. LN, $n=10$, $H_{0}: \sigma=\sigma_{0}=1$ Table entries are percentage.

\begin{tabular}{ccccccccc} 
Nominal size & $1 \%$ & $2.5 \%$ & $5 \%$ & $10 \%$ & $90 \%$ & $95 \%$ & $97.5 \%$ & $99 \%$ \\
\hline$\Phi(R)$ & 2.95 & 6.37 & 11.02 & 19.18 & 95.07 & 97.89 & 99.14 & 99.62 \\
$\Phi\left(R^{\prime}\right)$ & 8.36 & 12.74 & 18.37 & 26.24 & 91.58 & 95.03 & 96.95 & 98.35 \\
$\Phi\left(R^{*}\right)$ & 0.99 & 2.47 & 5.08 & 10.12 & 90.12 & 95.19 & 97.79 & 99.22 \\
Null hyp., $R^{\prime}$ & 0.92 & 2.32 & 4.69 & 9.63 & 89.96 & 95.19 & 97.82 & 99.13 \\
W. v1, $R^{\prime}(1.62 \%)$ & 6.07 & 9.99 & 14.23 & 20.57 & 89.95 & 95.26 & 97.84 & 99.21 \\
W. v2, $R^{\prime}(3.81 \%)$ & 5.21 & 7.14 & 10.13 & 15.19 & 89.79 & 95.00 & 97.62 & 99.20
\end{tabular}

It can be shown that the Kullback-Leibler distance is minimised when $\eta_{0}=\left(\sigma_{0}, \mu_{0}\right)$, where $\mu_{0}=\int(\log y) g(y) d y$ and $\sigma_{0}=\left\{\int\left(\log y-\mu_{0}\right)^{2} g(y) d y\right\}^{1 / 2}$, which may be calculated numerically. Then, the null hypothesis is set to be $H_{0}: \sigma=\sigma_{0}$.

The overall MLE of $\eta=(\sigma, \mu)$ is

$$
\hat{\eta}=(\hat{\sigma}, \hat{\mu})=\left(\left\{\frac{1}{n} \sum\left(\log Y_{i}-\hat{\mu}\right)^{2}\right\}^{1 / 2}, \frac{1}{n} \sum \log Y_{i}\right),
$$

and the constrained MLE of $\eta$ is $\hat{\eta}_{0}=\left(\sigma_{0}, \frac{1}{n} \sum \log Y_{i}\right)$. As can be seen from Table 3, the null hypothesis bootstrap procedure again performs better than all of the other approximation methods, even though the actual sizes are more different from the nominal sizes in the lower tail than the corresponding figures seen in the previous example. At $n=10$, the distributions of all the statistics, most particularly $R^{\prime}$, are very far from $N(0,1)$ : the normal approximation results in the table are poor. Of course, as $n$ increases, $R^{\prime}$ will tend in distribution to $N(0,1)$, whereas $R$ and $R^{*}$ will not. When $Y_{1}, \ldots, Y_{n}$ are actually from the assumed log-normal distribution with $\sigma_{0}=1$ and $\mu_{0}=1$, the normal approximation to $R^{*}$ and the null hypothesis bootstrap procedure applied with $R^{\prime}$ perform equally well and give the most 
effective approximations, as exhibited in Table 4. It can be observed that the normal approximation to $R^{\prime}$ performs least effectively, as in the previous example, under no model mis-specification.

Example 3. Logistic v.s. Gaussian. Let $Y_{1}, \ldots, Y_{n}$ be a sample from a logistic distribution with mean parameter $a=0$ and scale parameter $s=1$. Suppose the sample is assumed to be from a Gaussian distribution with mean $\mu$ and variance $\sigma^{2}$, and the parameter of interest is $\sigma^{2}$.

It can be calculated that $\eta_{0}=\left(\sigma_{0}^{2}, \mu_{0}\right)=\left(\frac{1}{3} \pi^{2} s^{2}, a\right)$ minimises the KullbackLeibler distance between the two distributions and therefore the null hypothesis is set to be $H_{0}: \sigma^{2}=\sigma_{0}^{2}$.

The overall MLE of $\eta=\left(\sigma^{2}, \mu\right)$ under the assumed distribution is $\hat{\eta}=$ $\left(\hat{\sigma}^{2}, \hat{\mu}\right)=\left(\frac{1}{n} \sum\left(Y_{i}-\hat{\mu}\right)^{2}, \frac{1}{n} \sum Y_{i}\right)$, and the constrained MLE of $\eta$ is $\hat{\eta}_{0}=$ $\left(\sigma_{0}^{2}, \frac{1}{n} \sum Y_{i}\right)$.

Table 5: Actual sizes of test under model mis-specification: Logistic v.s. Gaussian, $n=10$, $H_{0}: \sigma^{2}=\sigma_{0}^{2}$. Table entries are percentage.

\begin{tabular}{ccccccccc} 
Nominal size & $1 \%$ & $2.5 \%$ & $5 \%$ & $10 \%$ & $90 \%$ & $95 \%$ & $97.5 \%$ & $99 \%$ \\
\hline$\Phi(R)$ & 5.17 & 10.01 & 16.31 & 25.36 & 92.23 & 95.36 & 97.31 & 98.53 \\
$\Phi\left(R^{\prime}\right)$ & 12.00 & 17.37 & 23.23 & 31.80 & 91.05 & 94.52 & 96.40 & 97.87 \\
$\Phi\left(R^{*}\right)$ & 2.14 & 4.58 & 8.03 & 15.43 & 87.47 & 92.41 & 95.24 & 97.44 \\
Null hyp., $R^{\prime}$ & 1.65 & 4.08 & 7.35 & 13.57 & 89.44 & 94.68 & 97.19 & 98.85 \\
W. v1, $R^{\prime}(2.16 \%)$ & 11.20 & 15.90 & 20.54 & 27.73 & 90.25 & 95.32 & 97.58 & 99.10 \\
W. v2, $R^{\prime}(6.24 \%)$ & 7.52 & 9.41 & 12.74 & 18.34 & 89.83 & 94.78 & 97.06 & 98.73
\end{tabular}

Table 6: Actual sizes of test under no model mis-specification: Gaussian v.s. Gaussian, $n=10, H_{0}: \sigma^{2}=\sigma_{0}^{2}=4$. Table entries are percentage.

\begin{tabular}{ccccccccc} 
Nominal size & $1 \%$ & $2.5 \%$ & $5 \%$ & $10 \%$ & $90 \%$ & $95 \%$ & $97.5 \%$ & $99 \%$ \\
\hline$\Phi(R)$ & 3.04 & 6.32 & 10.97 & 19.08 & 94.75 & 97.86 & 99.06 & 99.62 \\
$\Phi\left(R^{\prime}\right)$ & 9.01 & 13.36 & 18.81 & 26.40 & 91.44 & 94.77 & 96.73 & 98.18 \\
$\Phi\left(R^{*}\right)$ & 1.20 & 2.64 & 5.02 & 10.31 & 90.03 & 94.98 & 97.66 & 99.11 \\
Null hyp., $R^{\prime}$ & 0.88 & 2.43 & 4.98 & 10.24 & 89.97 & 94.85 & 97.48 & 99.05 \\
W. v1, $R^{\prime}(1.40 \%)$ & 9.12 & 13.10 & 17.37 & 23.82 & 90.27 & 95.19 & 97.58 & 99.09 \\
W. v2, $R^{\prime}(3.96 \%)$ & 5.55 & 7.49 & 10.82 & 15.81 & 89.99 & 94.76 & 97.28 & 98.95
\end{tabular}

In Table 5, it is seen again that null hypothesis bootstrap approximation to the distribution of $R^{\prime}$ yields tests of actual sizes closest to the nominal sizes under model mis-specification. On the other hand, normal approximation to 
$R^{\prime}$ appears to be the most inaccurate distributional approximation. The distribution of $R^{\prime}$ at $n=10$ is far from standard normal, while normal approximation to $R^{*}$ seems to do rather well in this context.

If $Y_{1}, \ldots, Y_{n}$ are indeed from the Gaussian distribution with $\sigma_{0}^{2}=4$ and $\mu_{0}=1$, the use of the statistic $R^{\prime}$ with the null hypothesis bootstrap procedure and normal approximation to $R^{*}$ provide the best approximations, as seen in Table 6.

Example 4. Log-normal regression (LNRe) v.s. Exponential regression (ExpRe). Let $Y_{1}, \ldots, Y_{n}$ be a sample from a log-normal distribution having mean-log parameter $x_{1} \mu$ and variance-log parameter $x_{2} \sigma^{2}$, where $x_{1}, x_{2}$ are observed values from two independent variables each following a uniform distribution with the support $[0,1], \mu=-0.7$ and $\sigma=1$. Suppose the sample is assumed to be from an exponential distribution with mean $\frac{1}{\tau} \exp \left(\nu x_{1}+\xi x_{2}\right)$, where $x_{1}, x_{2}$ have the same definitions as before. This provides a threeparameter example with $\eta=(\nu, \xi, \tau)$, where $\nu$ will be taken as the parameter of interest, and $(\xi, \tau)$ is a vector nuisance parameter.

Denote by $\eta_{0}=\left(\nu_{0}, \xi_{0}, \tau_{0}\right), \hat{\eta}=(\hat{\nu}, \hat{\xi}, \hat{\tau})$ and $\hat{\eta}_{0}=\left(\nu_{0}, \hat{\xi}_{0}, \hat{\tau}_{0}\right)$ respectively the value of $\eta$ which minimises the Kullback-Leibler distance, the overall MLE of $\eta$ and the constrained MLE of $\eta$ for fixed $\nu_{0}$. Here, it can be derived that $\hat{\tau}=\frac{1}{n} \sum Y_{i} \exp \left(x_{1 i} \hat{\nu}+x_{2 i} \hat{\xi}\right)$ and $\hat{\tau}_{0}=\frac{1}{n} \sum Y_{i} \exp \left(x_{1 i} \nu_{0}+x_{2 i} \hat{\xi}_{0}\right)$, where $x_{1 i}$ and $x_{2 i}$ are the observed $x_{1}$ and $x_{2}$ values associated with $Y_{i}$. There are no closed forms for all other quantities in $\eta_{0}, \hat{\eta}$ and $\hat{\eta}_{0}$, but they can be easily computed numerically. The null hypothesis is set to be $H_{0}: \nu=\nu_{0}$.

The $U$ quantity required by the definition of Barndorff-Nielsen's $R^{*}$ statistic is difficult to construct in this example. Therefore, an estimate $\hat{U}$ of $U$ is used to construct an approximation, which is denoted by $\hat{R}^{*}=R+$ $\log (\hat{U} / R) / R$. The statistic $\hat{R}^{*}$ follows, under the null hypothesis, a standard normal distribution to $O_{p}\left(n^{-1}\right)$ under no model mis-specification; more details can be found in Severini (2000, Section 7.5.5).

As noted, another difference to the previous three examples is that the non-parametric bootstrap methods used are a mean adjustment version of the weighted resampling (w.m.a.). That is, the bootstrap values are centred at the mean of these bootstrap statistic values, as was described in Step 2 in Section 4.

As can be seen from Table 7, the approximation results obtained from the null hypothesis bootstrap method using the statistic $R^{\prime}$ are the closest to nominal sizes, which means the parametric method is to be viewed again 
Table 7: Actual sizes of test under model mis-specification: LNRe v.s. ExpRe, $n=10$, $H_{0}: \nu=\nu_{0}$. Table entries are percentage.

\begin{tabular}{ccccccccc} 
Nominal size & $1 \%$ & $2.5 \%$ & $5 \%$ & $10 \%$ & $90 \%$ & $95 \%$ & $97.5 \%$ & $99 \%$ \\
\hline$\Phi(R)$ & 0.18 & 0.53 & 1.29 & 3.96 & 96.56 & 98.71 & 99.57 & 99.90 \\
$\Phi\left(R^{\prime}\right)$ & 7.71 & 10.94 & 14.74 & 20.53 & 79.91 & 85.04 & 88.94 & 92.39 \\
$\Phi\left(\hat{R}^{*}\right)$ & 0.70 & 1.45 & 2.98 & 6.13 & 94.04 & 97.23 & 98.52 & 99.09 \\
Null hyp., $R^{\prime}$ & 0.92 & 2.35 & 4.83 & 10.10 & 89.95 & 95.10 & 97.65 & 99.08 \\
W.m.a. v1, $R^{\prime}(4.82 \%)$ & 1.19 & 2.57 & 5.12 & 10.23 & 98.03 & 99.77 & 99.84 & 99.87 \\
W.m.a. v2, $R^{\prime}(5.01 \%)$ & 0.57 & 0.86 & 2.07 & 7.27 & 97.32 & 99.93 & 100.00 & 100.00
\end{tabular}

as the favoured approach. Also, as in the previous three examples, normal approximation to $R^{\prime}$ does not seem to be satisfactory at the small sample size $n$ considered, and neither does the weighted v2. Weighted v1 does rather well in the lower tail. Normal approximation to $R$ and $\hat{R}^{*}$ appears to perform reasonably, due to the fact that the distributions of $R$ and $\hat{R}^{*}$ are not too different from $N(0,1)$ for the case considered; however, as the sample size increases, accuracy of these normal approximations will not improve. The

Table 8: Actual sizes of test under no model mis-specification: ExpRe v.s. ExpRe, $n=10$, $H_{0}: \nu=\nu_{0}=1.5$. Table entries are percentage.

\begin{tabular}{ccccccccc} 
Nominal size & $1 \%$ & $2.5 \%$ & $5 \%$ & $10 \%$ & $90 \%$ & $95 \%$ & $97.5 \%$ & $99 \%$ \\
\hline$\Phi(R)$ & 1.26 & 3.10 & 5.62 & 10.66 & 89.51 & 94.35 & 97.13 & 98.80 \\
$\Phi\left(R^{\prime}\right)$ & 7.84 & 11.10 & 14.91 & 20.37 & 80.35 & 85.72 & 89.09 & 92.33 \\
$\Phi\left(\hat{R}^{*}\right)$ & 1.00 & 2.56 & 5.04 & 10.33 & 90.64 & 95.22 & 97.51 & 98.92 \\
Null hyp., $R^{\prime}$ & 1.16 & 2.63 & 5.19 & 10.12 & 90.00 & 94.98 & 97.47 & 98.98 \\
W.m.a. v1, $R^{\prime}(6.30 \%)$ & 1.22 & 2.49 & 4.89 & 10.01 & 97.81 & 99.56 & 99.77 & 99.80 \\
W.m.a. v2, $R^{\prime}(5.46 \%)$ & 0.57 & 0.88 & 2.12 & 6.88 & 97.60 & 99.93 & 100.00 & 100.00
\end{tabular}

approximation results under model correctness, namely when the true distribution of $Y_{1}, \ldots, Y_{n}$ is the assumed exponential regression distribution with $\nu_{0}=1.5, \xi_{0}=0.9$ and $\tau_{0}=0.5$, are given in Table 8. Normal approximation to the distribution of $\hat{R}^{*}$ and parametric bootstrapping of $R^{\prime}$ work very well, as expected. The normal approximation to $R$ also performs well but the normal approximation to $R^{\prime}$ performs very poorly. The non-parametric bootstrapping of $R^{\prime}$ with the mean adjustment (w.m.a v2) does not seem to work very well at $n=10$ but it should be noted that results at moderate sample sizes such as $n=50$ are as good as those for the parametric alternative (see Table 17 in the Appendix). 


\section{Conclusions}

It can be seen from the examples presented that when there is no model mis-specification, the normal approximation to $R^{*}$ and the parametric bootstrap approximation to $R^{\prime}$ are very accurate, even for small sample size $n$, while the normal approximations to $R$ and especially $R^{\prime}$ perform less well. In addition, the non-parametric approximations to the distribution of $R^{\prime}$ does not appear to yield tests of size close to the desired nominal size either.

On the other hand, when the true distribution underlying the sample is mis-specified, the use of the parametric bootstrap method with $R^{\prime}$ provides the best approximation results, compared to normal approximations to $R$, $R^{\prime}$ and $R^{*}$, or the non-parametric methods applied with $R^{\prime}$. The reason why the normal approximations to $R$ and $R^{*}$ are not accurate is that the distributions of both statistics are not $N(0,1)$ asymptotically, with the finite sample distributions being far from $N(0,1)$. In contrast, although the asymptotic distribution of $R^{\prime}$ is $N(0,1)$, the distribution of $R^{\prime}$ can be very different from standard normal for small $n$, and it typically only gradually approaches $N(0,1)$ as $n$ increases.

The examples have shown that, applied with the statistic $R^{\prime}$, the parametric bootstrap method, which involves simulating from the correctly assumed distribution when $g=f$, or wrongly assumed distribution when $g \neq f$, achieves the best approximation results of the approximation methods considered. The robust statistic $R^{\prime}$ is chosen here to guard against possible model mis-specification, while the use of the parametric bootstrap method ensures that it is not necessary to be concerned with efficiency loss, as discussed by Stafford (1996), when $g=f$, since the parametric bootstrap procedure works equally well with $R$ and $R^{\prime}$ in this situation.

The key reason for using $R$ ' is that the statistic is more 'stable' in comparison to $R$ or $R^{*}$. Regardless of how wrong the assumed distribution may be, as measured by the Kullback-Leibler distance between $g$ and $f$, the distribution of $R^{\prime}$ is typically rather similar. However, the distributions of $R$ or $R^{*}$ can change dramatically. The robustification of the signed root statistic is designed to yield a statistic $R^{\prime}$ which is asymptotically standard normal, even under model mis-specification. But the modification is seen to lead to a statistic which, even for small sample size $n$, has a distribution which varies only very slightly with change in the true underlying distribution $g$, which allows for effective estimation of its distribution by simulation from an incorrect model. Figure 1 demonstrates graphically what is meant by the 

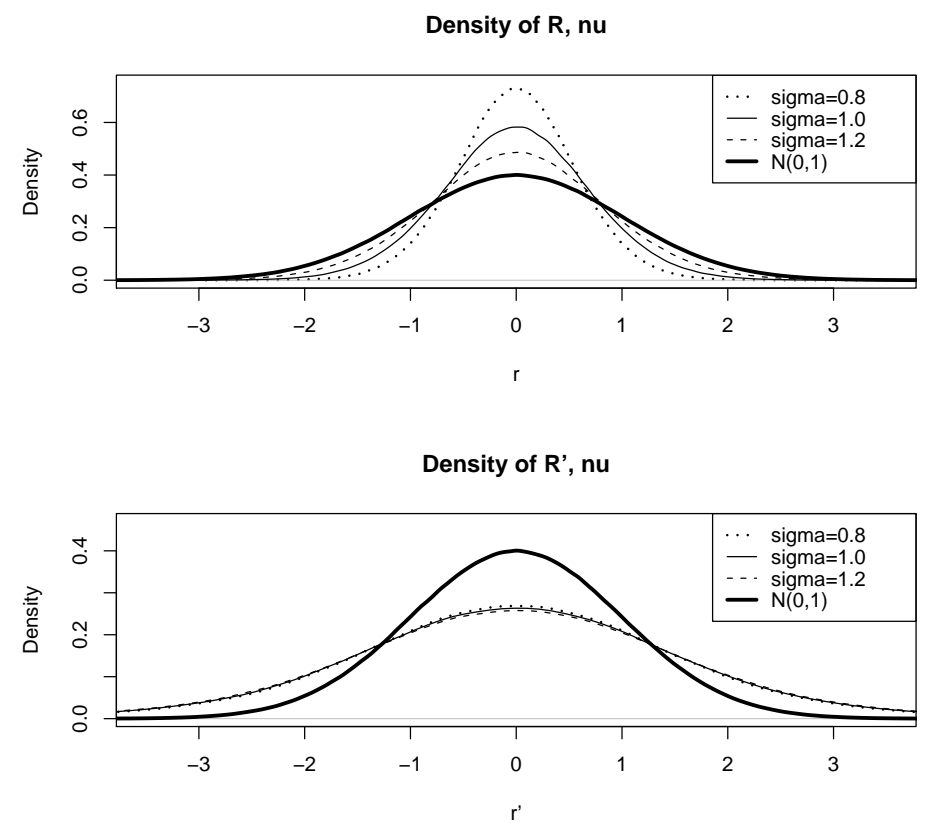

Figure 1: LnRe v.s.ExpRe, $\nu$ : densities of $R$ (top) and $R^{\prime}$ (bottom) for different $g, n=10$

stability of a statistic. When the $\sigma$ parameter of the log-normal regression distribution increases from 0.8 to 1.2 , the Kullback-Leibler distance between the log-normal regression distribution and the assumed exponential regression distribution changes, and it can be seen that the density plot of $R$ varies much more than the density plot of $R^{\prime}$.

The same trend can be seen in the further examples of density plots for $R$ and $R^{\prime}$ in Figure 2, relating to Example 1. Note that now the change in distribution of $R$ due to the change (of the parameter $c$ ) in the true underlying distribution seems to be smaller than that observed in the previous case. Density plots of $R^{*}$ show similar features to $R$ and are not included here. Both Figures 1 and 2 show that the distribution of $R$ (for the cases $\sigma=1$ and $c=5.5$ examined above) appears to be closer to the standard normal distribution than is the distribution of $R^{\prime}$, which explains why the normal approximation to $R$ can work better than the normal approximation to $R^{\prime}$ in some situations.

Results from further simulations with moderate sample size $n=50$ show that $R$ and $R^{*}$ are not distributed as close to $N(0,1)$, as can be seen from the 

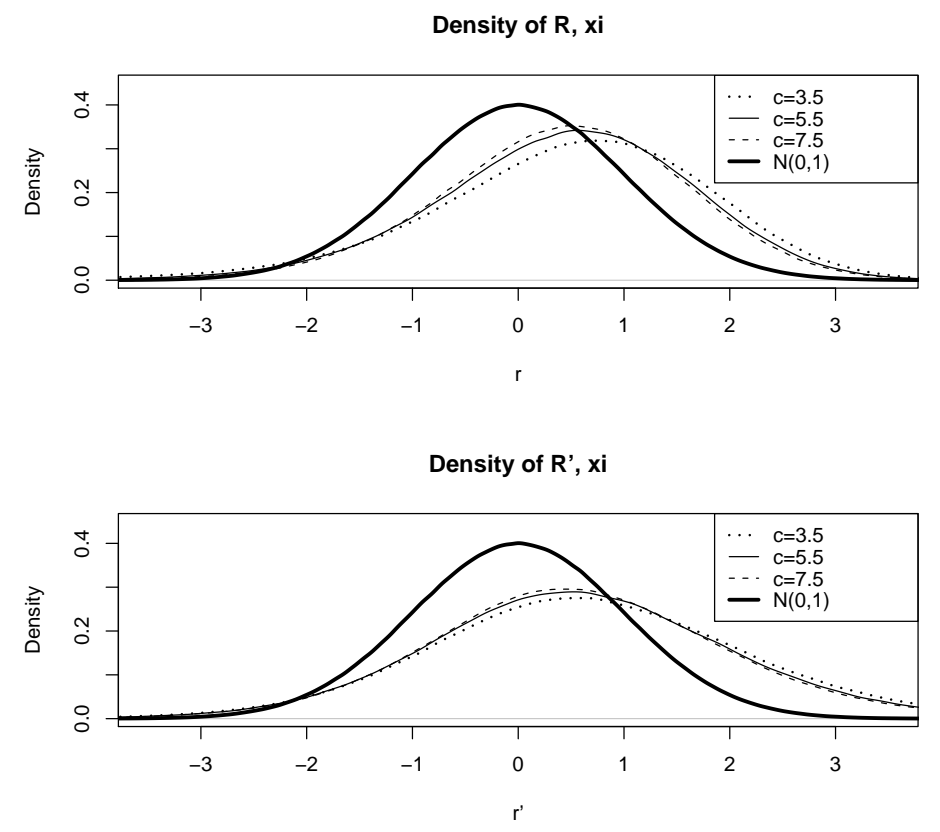

Figure 2: Gamma v.s. IG, $\xi$ : densities of $R$ (top) and $R^{\prime}$ (bottom) for different $g, n=10$

worse results from normal approximation to the distributions of $R$ and $R^{*}$ in Tables 10, 12, 14 and 16 in the Appendix. In contrast, the results from the use of the $R^{\prime}$ statistic become much closer to their respective nominal values as $n$ increases, compared to what is observed for $n=10$. When there is no model mis-specification, Tables 11, 13, 15 and 17 show that results from all the methods become highly accurate: note in particular the marked improvement in both weighted v1 and weighted v2 methods. Another observation from the examples, which has already been remarked upon, is that results for the ad hoc weighted v2 method are generally quite similar to or better than the results for the more sophisticated weighted v1 method, whether $g=f$ or $g \neq f$. Again, however, it should be noted that results from non-parametric sampling are generally inferior to those of the parametric bootstrap scheme, of particular interest being the case where there is model mis-specification.

Finally, it is seen from Table 9 that, as $n$ increases, empirical estimates of the cumulants of $R, R^{\prime}$ and $R^{*}$, as constructed from a large simulation, all tend to the cumulants of $N(0,1)$ when $g=f$ in Example 3. As all examples exhibit identical characteristics, only results for this example are included 
Table 9: Empirical estimates of the first four cumulants $\kappa_{1}, \kappa_{2}, \kappa_{3}$ and $\kappa_{4}$ of $R, R^{\prime}$ and $R^{*}, B=10^{6}$.

\begin{tabular}{r|rrrrr|rrrrrr} 
& \multicolumn{6}{|c|}{ Gaussian v.s. Gaussian, $x$} & \multicolumn{6}{|c}{ Logistic v.s. Gaussian, $x$} \\
\hline & $n$ & $\kappa_{1}$ & $\kappa_{2}$ & $\kappa_{3}$ & $\kappa_{4}$ & $n$ & $\kappa_{1}$ & $\kappa_{2}$ & $\kappa_{3}$ & $\kappa_{4}$ \\
\hline$R$ & 10 & -0.3933 & 1.0495 & -0.0060 & 0.0038 & 10 & -0.4584 & 1.4443 & 0.5071 & 0.5960 \\
& 50 & -0.1691 & 1.0101 & 0.0019 & 0.0084 & 100 & -0.1474 & 1.5750 & 0.2746 & 0.2009 \\
& 100 & -0.1183 & 1.0038 & 0.0016 & -0.0036 & 500 & -0.0667 & 1.5903 & 0.1346 & 0.0497 \\
\hline$R^{\prime}$ & 10 & -0.5069 & 1.8735 & -0.4192 & 3.4563 & 10 & -0.6241 & 2.2140 & -0.5964 & 3.7665 \\
& 50 & -0.1801 & 1.1700 & -0.0225 & 0.3421 & 100 & -0.1808 & 1.1895 & -0.2057 & 0.2686 \\
& 100 & -0.1223 & 1.0869 & -0.0069 & 0.1596 & 500 & -0.0841 & 1.0481 & -0.1153 & 0.0729 \\
\hline$R^{*}$ & 10 & -0.0104 & 1.0014 & 0.0026 & 0.0083 & 10 & -0.0735 & 1.3789 & 0.4904 & 0.5817 \\
& 50 & -0.0016 & 1.0012 & 0.0027 & 0.0107 & 100 & -0.0291 & 1.5681 & 0.2736 & 0.2004 \\
& 100 & -0.0002 & 0.9994 & 0.0013 & 0.0004 & 500 & -0.0139 & 1.5889 & 0.1342 & 0.0506
\end{tabular}

here. The same clear trend is only observed, in particular for the second cumulant $\kappa_{2}$, under model mis-specification, $g \neq f$, for the cumulants of $R^{\prime}$. Note, however, a slow convergence rate, indicating that $R^{\prime}$ approaches its asymptotic $N(0,1)$ limit rather slowly, while $R$ and $R^{*}$ are not asymptotically standard normal in distribution under model mis-specification.

\section{Appendix}

Table 10: Actual sizes of test under model mis-specification: Gamma v.s. IG, $n=50$, $H_{0}: \xi=\xi_{0}$. Table entries are percentage.

\begin{tabular}{ccccccccc} 
Nominal size & $1 \%$ & $2.5 \%$ & $5 \%$ & $10 \%$ & $90 \%$ & $95 \%$ & $97.5 \%$ & $99 \%$ \\
\hline$\Phi(R)$ & 2.81 & 5.03 & 7.55 & 12.09 & 80.47 & 88.31 & 93.08 & 96.87 \\
$\Phi\left(R^{\prime}\right)$ & 1.02 & 2.20 & 4.32 & 8.55 & 81.54 & 88.88 & 93.08 & 96.22 \\
$\Phi\left(R^{*}\right)$ & 3.66 & 6.16 & 9.31 & 14.75 & 84.64 & 91.15 & 95.16 & 97.67 \\
Null hyp., $R^{\prime}$ & 0.93 & 2.32 & 4.99 & 9.97 & 87.23 & 93.01 & 96.26 & 98.35 \\
W. v1, $R^{\prime}(0 \%)$ & 0.84 & 2.27 & 4.84 & 9.80 & 84.08 & 90.00 & 93.67 & 96.27 \\
W. v2, $R^{\prime}(0 \%)$ & 0.93 & 2.41 & 5.01 & 9.92 & 84.34 & 90.19 & 93.93 & 96.47
\end{tabular}


Table 11: Actual sizes of test under no model mis-specification: IG v.s. IG, $n=50$, $H_{0}: \xi=\xi_{0}=2$. Table entries are percentage.

\begin{tabular}{ccccccccc} 
Nominal size & $1 \%$ & $2.5 \%$ & $5 \%$ & $10 \%$ & $90 \%$ & $95 \%$ & $97.5 \%$ & $99 \%$ \\
\hline$\Phi(R)$ & 0.60 & 1.83 & 3.80 & 7.53 & 87.10 & 93.01 & 96.11 & 98.20 \\
$\Phi\left(R^{\prime}\right)$ & 1.17 & 2.57 & 4.64 & 8.75 & 85.44 & 91.78 & 94.91 & 97.35 \\
$\Phi\left(R^{*}\right)$ & 0.97 & 2.73 & 5.21 & 10.47 & 90.31 & 95.05 & 97.16 & 98.99 \\
Null hyp., $R^{\prime}$ & 1.05 & 2.69 & 5.17 & 10.43 & 90.34 & 94.96 & 97.29 & 98.83 \\
W. v1, $R^{\prime}(0 \%)$ & 0.99 & 2.43 & 5.09 & 10.22 & 87.90 & 93.05 & 95.50 & 97.50 \\
W. v2, $R^{\prime}(0 \%)$ & 1.01 & 2.58 & 5.09 & 10.34 & 88.05 & 93.17 & 95.72 & 97.65
\end{tabular}

Table 12: Actual sizes of test under model mis-specification: F v.s. LN, $n=50, H_{0}: \sigma=$ $\sigma_{0}$. Table entries are percentage.

\begin{tabular}{ccccccccc} 
Nominal size & $1 \%$ & $2.5 \%$ & $5 \%$ & $10 \%$ & $90 \%$ & $95 \%$ & $97.5 \%$ & $99 \%$ \\
\hline$\Phi(R)$ & 6.28 & 10.73 & 15.84 & 23.95 & 85.87 & 90.44 & 93.28 & 95.51 \\
$\Phi\left(R^{\prime}\right)$ & 5.53 & 9.07 & 13.51 & 20.66 & 91.51 & 95.43 & 97.69 & 98.86 \\
$\Phi\left(R^{*}\right)$ & 4.52 & 8.18 & 12.88 & 19.83 & 83.39 & 88.56 & 91.93 & 94.67 \\
Null hyp., $R^{\prime}$ & 3.10 & 5.53 & 9.14 & 15.39 & 90.02 & 94.97 & 97.58 & 98.98 \\
W. v1, $R^{\prime}(0 \%)$ & 5.42 & 8.44 & 12.45 & 18.32 & 90.46 & 95.23 & 97.71 & 99.11 \\
W. v2, $R^{\prime}(0 \%)$ & 5.09 & 8.01 & 11.96 & 17.98 & 89.97 & 94.79 & 97.40 & 98.83
\end{tabular}

Table 13: Actual sizes of test under no model mis-specification: LN v.s. LN, $n=50$, $H_{0}: \sigma=\sigma_{0}=1$ Table entries are percentage.

\begin{tabular}{ccccccccc} 
Nominal size & $1 \%$ & $2.5 \%$ & $5 \%$ & $10 \%$ & $90 \%$ & $95 \%$ & $97.5 \%$ & $99 \%$ \\
\hline$\Phi(R)$ & 1.56 & 3.84 & 7.13 & 13.12 & 91.79 & 96.08 & 98.10 & 99.23 \\
$\Phi\left(R^{\prime}\right)$ & 2.46 & 5.04 & 8.68 & 14.80 & 90.85 & 94.96 & 97.36 & 98.81 \\
$\Phi\left(R^{*}\right)$ & 1.01 & 2.46 & 5.08 & 10.10 & 89.47 & 94.38 & 97.19 & 98.86 \\
Null hyp., $R^{\prime}$ & 1.04 & 2.49 & 5.02 & 10.17 & 89.48 & 94.42 & 97.20 & 98.88 \\
W. v1, $R^{\prime}(0 \%)$ & 2.39 & 4.55 & 7.55 & 12.54 & 89.66 & 94.58 & 97.33 & 99.00 \\
W. v2, $R^{\prime}(0 \%)$ & 2.19 & 4.39 & 7.37 & 12.38 & 89.55 & 94.45 & 97.26 & 98.97
\end{tabular}

Table 14: Actual sizes of test under model mis-specification: Logistic v.s. Gaussian, $n=50, H_{0}: \sigma^{2}=\sigma_{0}^{2}$. Table entries are percentage.

\begin{tabular}{ccccccccc} 
Nominal size & $1 \%$ & $2.5 \%$ & $5 \%$ & $10 \%$ & $90 \%$ & $95 \%$ & $97.5 \%$ & $99 \%$ \\
\hline$\Phi(R)$ & 3.80 & 7.09 & 11.87 & 19.53 & 88.90 & 92.96 & 95.68 & 97.64 \\
$\Phi\left(R^{\prime}\right)$ & 4.03 & 7.04 & 11.32 & 17.80 & 91.96 & 95.87 & 97.87 & 99.07 \\
$\Phi\left(R^{*}\right)$ & 2.70 & 5.56 & 8.91 & 15.45 & 86.35 & 91.34 & 94.50 & 96.86 \\
Null hyp., $R^{\prime}$ & 1.74 & 4.02 & 7.06 & 12.70 & 90.42 & 95.31 & 97.80 & 99.18 \\
W. v1, $R^{\prime}(0 \%)$ & 3.83 & 6.39 & 9.84 & 15.20 & 90.47 & 95.45 & 97.82 & 99.22 \\
W. v2, $R^{\prime}(0 \%)$ & 3.72 & 6.20 & 9.63 & 15.13 & 90.40 & 95.35 & 97.74 & 99.21
\end{tabular}


Table 15: Actual sizes of test under no model mis-specification: Gaussian v.s. Gaussian, $n=50, H_{0}: \sigma^{2}=\sigma_{0}^{2}=4$. Table entries are percentage.

\begin{tabular}{ccccccccc} 
Nominal size & $1 \%$ & $2.5 \%$ & $5 \%$ & $10 \%$ & $90 \%$ & $95 \%$ & $97.5 \%$ & $99 \%$ \\
\hline$\Phi(R)$ & 1.38 & 3.88 & 7.42 & 13.65 & 92.72 & 96.38 & 98.28 & 99.45 \\
$\Phi\left(R^{\prime}\right)$ & 2.35 & 5.14 & 9.18 & 15.25 & 91.53 & 95.60 & 97.51 & 99.05 \\
$\Phi\left(R^{*}\right)$ & 0.77 & 2.50 & 5.29 & 10.30 & 90.22 & 94.96 & 97.50 & 98.99 \\
Null hyp., $R^{\prime}$ & 0.87 & 2.36 & 5.17 & 10.59 & 90.22 & 95.00 & 97.44 & 99.15 \\
W. v1, $R^{\prime}(0 \%)$ & 2.26 & 4.61 & 7.94 & 12.92 & 90.52 & 95.13 & 97.63 & 99.20 \\
W. v2, $R^{\prime}(0 \%)$ & 2.11 & 4.30 & 7.79 & 12.82 & 90.32 & 95.21 & 97.49 & 99.20
\end{tabular}

Table 16: Actual sizes of test under model mis-specification: LNRe v.s. ExpRe, $n=50$, $H_{0}: \nu=\nu_{0}$. Table entries are percentage.

\begin{tabular}{ccccccccc} 
Nominal size & $1 \%$ & $2.5 \%$ & $5 \%$ & $10 \%$ & $90 \%$ & $95 \%$ & $97.5 \%$ & $99 \%$ \\
\hline$\Phi(R)$ & 0.18 & 0.74 & 1.96 & 5.10 & 94.92 & 98.16 & 99.39 & 99.85 \\
$\Phi\left(R^{\prime}\right)$ & 2.25 & 4.81 & 7.94 & 13.58 & 86.44 & 92.13 & 95.21 & 97.59 \\
$\Phi\left(\hat{R}^{*}\right)$ & 0.10 & 0.56 & 1.61 & 4.42 & 95.61 & 98.49 & 99.57 & 99.91 \\
Null hyp., $R^{\prime}$ & 0.83 & 2.49 & 5.44 & 10.52 & 89.43 & 94.65 & 97.23 & 99.12 \\
W.m.a. v1, $R^{\prime}(0 \%)$ & 1.51 & 3.36 & 6.43 & 11.15 & 88.85 & 93.80 & 96.32 & 98.29 \\
W.m.a. v2, $R^{\prime}(0 \%)$ & 1.13 & 2.95 & 5.96 & 11.21 & 88.66 & 93.91 & 96.67 & 98.78
\end{tabular}

Table 17: Actual sizes of test under no model mis-specification: ExpRe v.s. ExpRe, $n=50, H_{0}: \nu=\nu_{0}=1.5$. Table entries are percentage.

\begin{tabular}{ccccccccc} 
Nominal size & $1 \%$ & $2.5 \%$ & $5 \%$ & $10 \%$ & $90 \%$ & $95 \%$ & $97.5 \%$ & $99 \%$ \\
\hline$\Phi(R)$ & 1.15 & 2.44 & 5.18 & 10.16 & 89.92 & 95.01 & 97.52 & 99.09 \\
$\Phi\left(R^{\prime}\right)$ & 2.24 & 4.31 & 7.48 & 13.03 & 87.37 & 92.28 & 95.60 & 97.83 \\
$\Phi\left(\hat{R}^{*}\right)$ & 1.16 & 2.42 & 4.90 & 10.01 & 90.06 & 94.98 & 97.71 & 99.09 \\
Null hyp., $R^{\prime}$ & 1.09 & 2.44 & 4.92 & 10.14 & 89.68 & 95.02 & 97.59 & 99.11 \\
W.m.a. v1, $R^{\prime}(0 \%)$ & 1.57 & 3.27 & 5.86 & 11.18 & 89.00 & 94.07 & 96.83 & 98.67 \\
W.m.a. v2, $R^{\prime}(0 \%)$ & 0.98 & 2.43 & 4.86 & 10.41 & 89.30 & 94.79 & 97.42 & 99.10
\end{tabular}




\section{References}

Barndorff-Nielsen, O.E., 1986 Inference on full or partial parameters based on the standardized signed log likelihood ratio. Biometrika 73, 307-322.

DiCiccio, T.J., Martin, M.A., and Stern, S.E., 2001 Simple and accurate onesided inference from signed roots of likelihood ratios. Canadian Journal of Statistics 29, 67-76.

Kent, J.T., 1982 Robust properties of likelihood ratio tests. Biometrika 69, 19-27.

Lee, S.M.S. and Young, G.A., 2005 Parametric bootstrapping with nuisance parameters. Statistics \& Probability Letters 71, 143-153.

Lu, H.Y.K. and Young, G.A., 2010 Discussion of 'Maximum likelihood estimation of a multi-dimensional log-concave density', by Cule, Samworth \& Stewart. Journal Royal Statistical Society, Series B 72, 582-584.

Severini, T.A., 2000 Likelihood Methods in Statistics. Oxford University Press, Oxford.

Stafford, J.E., 1996 A robust adjustment of the profile likelihood. Annals of Statistics 24, 336-352.

Viraswami, K. and Reid, N., 1996 Higher-order asymptotics under model misspecification. Canadian Journal of Statistics 24, 263-278.

Viraswami, K. and Reid, N., 1998 A note on the likelihood ratio statistic under model misspecification. Canadian Journal of Statistics 26, 161-168.

Young, G. A., 2009 Routes to higher-order accuracy in parametric inference. Australian \& New Zealand Journal of Statistics 51, 115-126. 